\title{
Machine learning in cardiovascular magnetic resonance: basic concepts and applications
}

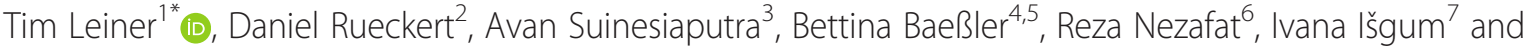 \\ Alistair A. Young ${ }^{3,8}$
}

\begin{abstract}
Machine learning (ML) is making a dramatic impact on cardiovascular magnetic resonance (CMR) in many ways. This review seeks to highlight the major areas in CMR where ML, and deep learning in particular, can assist clinicians and engineers in improving imaging efficiency, quality, image analysis and interpretation, as well as patient evaluation. We discuss recent developments in the field of ML relevant to CMR in the areas of image acquisition \& reconstruction, image analysis, diagnostic evaluation and derivation of prognostic information. To date, the main impact of ML in CMR has been to significantly reduce the time required for image segmentation and analysis. Accurate and reproducible fully automated quantification of left and right ventricular mass and volume is now available in commercial products. Active research areas include reduction of image acquisition and reconstruction time, improving spatial and temporal resolution, and analysis of perfusion and myocardial mapping. Although large cohort studies are providing valuable data sets for ML training, care must be taken in extending applications to specific patient groups. Since ML algorithms can fail in unpredictable ways, it is important to mitigate this by open source publication of computational processes and datasets. Furthermore, controlled trials are needed to evaluate methods across multiple centers and patient groups.
\end{abstract}

Keywords: Cardiovascular magnetic resonance, Machine learning, Deep learning, Radiomics

\section{Introduction}

Machine learning (ML) and artificial intelligence (AI) are rapidly gaining importance in medicine [1, 2], including in the field of medical imaging, and are likely to fundamentally transform clinical practice in the coming years [3, 4]. AI refers to the wider application of machines that perform tasks that are characteristic of human intelligence, e.g. infer conclusions from deduction or induction, while ML is a more restricted form of computational processing which uses a mathematical model together with training data to learn how to make predictions. Rather than explicitly computing results from a set of predefined rules, ML learns parameters from examples and therefore has the potential to perform better at a task such as detecting and

\footnotetext{
*Correspondence: T.Leiner@umcutrecht.nl

'Department of Radiology | E.01.132, Utrecht University Medical Center, Heidelberglaan 100, 3584CX Utrecht, The Netherlands

Full list of author information is available at the end of the article
}

differentiating patterns in data by being exposed to a more examples. The most advanced ML techniques, also called deep learning (DL), are especially well-suited for this purpose (Fig. 1). Cardiovascular magnetic resonance (CMR) is a field that lends itself to ML because it relies on complex acquisition strategies, including multidimensional contrast mechanisms, as well as the need for accurate and reliable segmentation and quantification of biomarkers based on acquired data, to help guide diagnosis and therapy management.

It is important for clinicians and researchers working in CMR to understand the impact of ML on the field. Thus, the purpose of this review is threefold: firstly, we will provide a non-technical overview of the basics of ML relevant to CMR. Secondly, we survey the various ways ML has been applied to the field of CMR. Finally, we provide an outlook on future directions and recommendations for reporting results. Please also refer the

(c) The Author(s). 2019 Open Access This article is distributed under the terms of the Creative Commons Attribution 4.0 International License (http://creativecommons.org/licenses/by/4.0/), which permits unrestricted use, distribution, and 


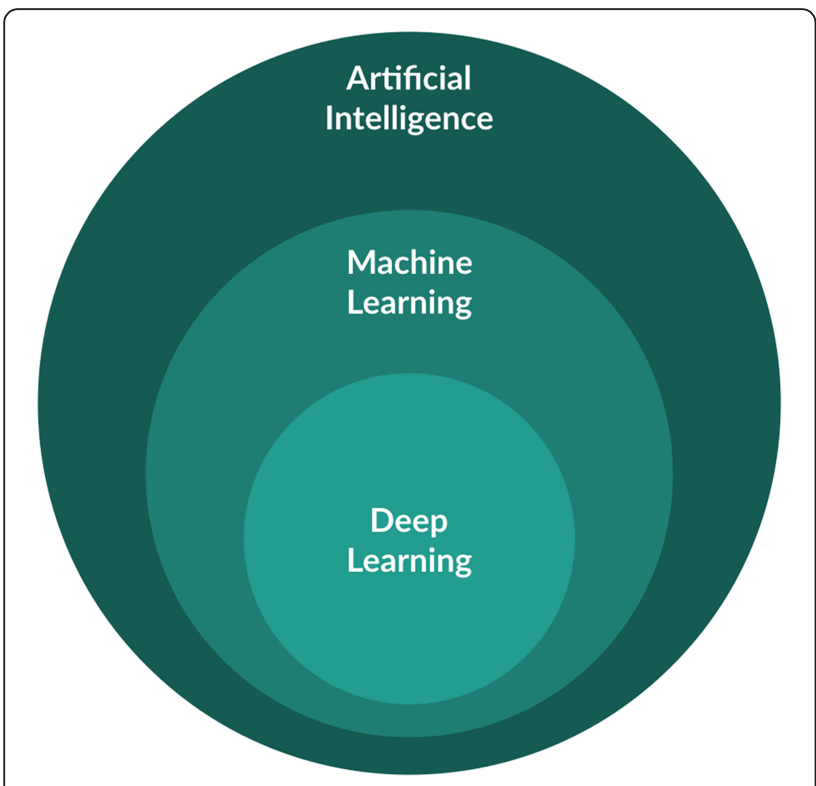

Fig. 1 Artificial intelligence (Al) can be seen as any technique that enables computers to perform tasks characteristic of human intelligence. Machine learning (ML) is generally seen as the subdiscipline of Al which uses a statistical model together with training data to learn how to make predictions. Deep learning (DL) is a specific form of ML that uses artificial neural networks with hidden layers to make predictions directly from datasets

glossary of terms for definitions of commonly used terms in machine learning.

\section{Machine learning basics}

Consider the problem of reporting left ventricular (LV) ejection fraction (LVEF) from a CMR study. A traditional image processing method would typically define a sequence of steps a priori, e.g. selection of end diastolic (ED) and end systolic (ES) frames, contouring of cavity and myocardium using signal processing algorithms with a sequence of processing steps, calculation of cavity area per slice, summation into volumes, and the calculation of LVEF. In comparison, a ML method would learn from a set of examples, e.g. hundreds of CMR studies with ground truth segmentations, to optimize a mathematical model which is then used to predict segmentations. In this case the algorithm learns which parts of the data are important for the task, and how to put the information together to produce the result.

\section{Standard machine learning models}

In standard ML model, important characteristics or features for performing a certain task are extracted from images by using a designed feature set. In the example above, features for myocardial contours may include image contrast, noise characteristics, texture and motion. Once the design process is complete, ML methods need to be trained using example data. In this training phase, parameters of the feature set model are learned. A model is any function of the features used for prediction and the parameters of the model dictate the actual predictions made. Once trained, the model can be used to make a prediction for data not seen previously in the training phase. ML models can perform either classification where discrete labels such as the presence or absence of disease are determined, or regression, where continuous variables such as $\mathrm{T} 1$ are estimated. Because the models learn from examples, it is important that a sufficiently large dataset with representative variability is available for training. For evaluation of the model's performance it is of utmost importance to keep training data that is used during model development and finetuning separated from the test data that is used to evaluate the model's performance. Another dataset (usually called the validation dataset), is used during the training phase to help determine the optimal design of the ML model. This dataset is used to optimize model parameters, and to ensure that the model does not overfit.

\section{Deep learning}

One of the key steps in creating ML systems is designing the optimal discriminative features for a given task. This has proven highly challenging $[5,6]$. A subfield of machine learning that can address this challenge is DL. Unlike standard ML methods, DL methods are able to learn directly from the data, circumventing the need for hand-crafting of discriminative features. In the example of finding the contours of myocardium, DL methods learn the image features most useful for predicting the location of the contours.

Recent successes with DL have been fueled by four synergistic advances: 1 ) the availability of large quantities of high-quality digital image data for training; 2) the ability of algorithms to learn relevant information directly from images without the need for handcrafted features; 3) low-cost powerful graphics processing unit (GPU) hardware, and 4) open source development libraries and working example networks made freely available by companies and researchers. These advances have led to the development of neural networks with many layers, which is what 'deep' refers to in DL [7]. A special type of DL network, the convolutional neural network (CNN) is often used for image analysis tasks.

A typical CNN network is composed of multiple layers, each with a well-defined architecture (Fig. 2). Convolution layers refer to those which employ a set of filters that are applied to the image to produce spatially dependent features for the next layer. The intent is to learn the optimal values of the filters (also called weights) so that features of maximum relevance to the task are generated in the subsequent layers. Pooling layers (e.G. max pooling or average pooling) downsample the spatial information so that 


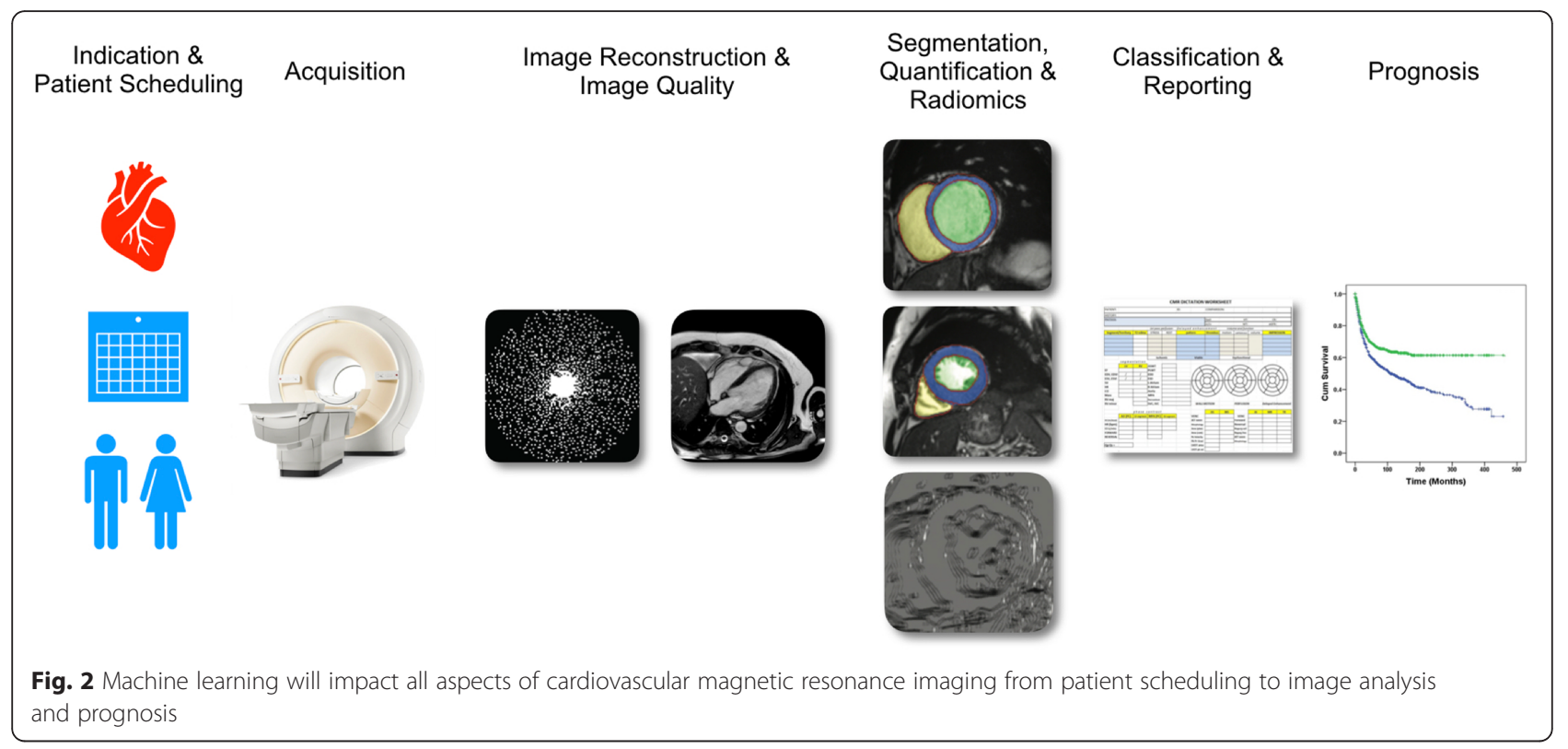

features become more canonical for the task. For classification and some regression networks, a fully connected layer is used in which each node is connected to all other nodes in the layer. Segmentation networks often use upsampling operations to return the image dimensions back to the input image size. Skip layers are often employed, which enable the propagation of fine details from one layer to another, with the intent of recovering fine imaging features and improving gradient propagation during training. Finally, a softmax layer performs a non-linear function which rescales the components to give a non-negative probability to each pixel class. This ensures that outputs sum up to 1 in the output layer. Often deep CNN implementations contain many millions of weights. Although the features resulting from the convolutions in the intermediate layers contain information pertinent to the task, it is often difficult to interpret how the network makes its predictions, or why it failed. However, DL is currently the most popular ML architecture for medical image analysis. A recent survey [8] shows more than 300 DL papers have been contributed to the medical image analysis field, including CMR, in the 6 years to 2017, with the numbers growing exponentially.

\section{Supervised and unsupervised learning}

Based on the availability of reference labels in the training data, ML algorithms are commonly divided into supervised and unsupervised learning. In supervised learning, training data are accompanied with ground truth labels, e.g. cases with pathological status, images with expert-drawn contours, or cases with cardiac volume and function measurements. Supervised learning is the most commonly used approach in ML because learning from expert-annotated labels is the most intuitive way to mimic human performance. This is in contrast to unsupervised learning where training data are given without labels. Unsupervised learning is more challenging for building a prediction model, because it is closer to natural learning by discovering structures through observation [7]. Currently, a typical use of unsupervised learning is to explore hidden structure inside the training data. An example relevant to CMR is the work by Oksuz et al. who used unsupervised dictionarybased learning to segment myocardium from cine blood oxygen level dependent (BOLD) CMR [9].

ML model parameters can be estimated by assuming a simple functional relationship between the data and the labels, for instance between CMR images and a certain diagnosis. A classic example is linear discriminant analysis, which learns to fit a hyperplane to the training data by optimizing linear coefficients, e.g. to separate patients with reduced LVEF from subjects with normal LVEF. However, typical problems are complex and multi-dimensional with a large amount of data, and simple mathematical relationships cannot be assumed. Alternatively, ML model parameters can be optimized by an iterative process designed to refine the model behavior under some regularization constraints. Regularization is a mathematical tool to take into account prior information when solving an optimization task. Examples are support vector machines, e.g. applied to characterize vessel disease from intravascular images [10], random forests e.g. applied for T2 map quantification [11]) and DL CNNs, which is the focus of this review.

\section{Current applications of machine learning in CMR Image acquisition and reconstruction}

Efficient, high-quality CMR demands careful attention to proper patient positioning as well as planning of imaging 
planes and volumes [12]. In current clinical practice, CMR examinations are therefore performed by highly experienced operators. Several of these acquisitionrelated aspects of the CMR examination, which are currently performed manually on most commercial CMR systems, can be either automated or substantially shortened using ML. Multiple CMR hardware vendors are working on workflow optimizations such fully automated localization of the heart and planning of image acquisition planes aligned with the principal cardiac axes $[13,14]$. Other investigators have applied ML to automate optimal frequency adjustment for CMR at $3 \mathrm{~T}$ [15], and to create a scan control framework that detects image artifacts during the scan and self-corrects imaging parameters or triggers a rescan if the prediction indicates the current slice has artifacts [16].

While CMR imaging offers a range of advantages for assessment of cardiac structure and function, acquisition of CMR images is slow as it is complicated by cardiac and respiratory motion. This imposes significant demands on patients (e.g. in terms of length of scan time and length of breath-holds) as well as making CMR expensive and less accessible. Over the last decade approaches such as parallel imaging and compressed sensing (CS) as well as realtime imaging have been increasingly employed to accelerate the acquisition of CMR images [17-26]. Techniques such as CS are particularly attractive for accelerating CMR as they undersample $\mathrm{k}$-space, thereby leading to faster image acquisition. CS techniques such as [27-29] can be regarded as ML methods that exploit spatiotemporal redundancies in CMR data to learn how to recover an uncorrupted image from undersampled k-space measurements. For this, CS techniques exploit the sparsity (or compressibility) of CMR images. More recently DL techniques have emerged that use convolutional neural networks in order to replace the generic sparsity model used in CS techniques with a model that is learnt from training data [30,31]. An advantage of these DL approaches is that they not only offer superior performance in terms of reconstruction quality but that they also offer high efficiency, e.g. very fast reconstruction speeds, making clinical deployment feasible [31] (Fig. 3).

More recently, DL approaches that exploit spatiotemporal redundancy via recurrent $\mathrm{CNNs}$ have been proposed which are more compact than cascades of CNNs [32]. A remaining challenge is the integration of DL approaches with existing approaches for the acceleration of CMR, such as parallel or real-time imaging. Accelerated imaging is necessary in high-dimensional (e.g. 3D or 4D) imaging for late gadolinium enhancement (LGE), flow or perfusion imaging. DL techniques have the potential to be applied to reduce the reconstruction time of highly accelerated 3D or 4D dataset. Figure 4 shows an example 3D LGE image reconstructed using DL with an acceleration factor of 5 .

\section{Image segmentation}

Delineating the borders of the chambers and myocardium (a process known as segmentation) is mandatory in CMR post processing [33], but it is a time-consuming task. Experienced readers may produce high precision manual contours, but differences among expert readers

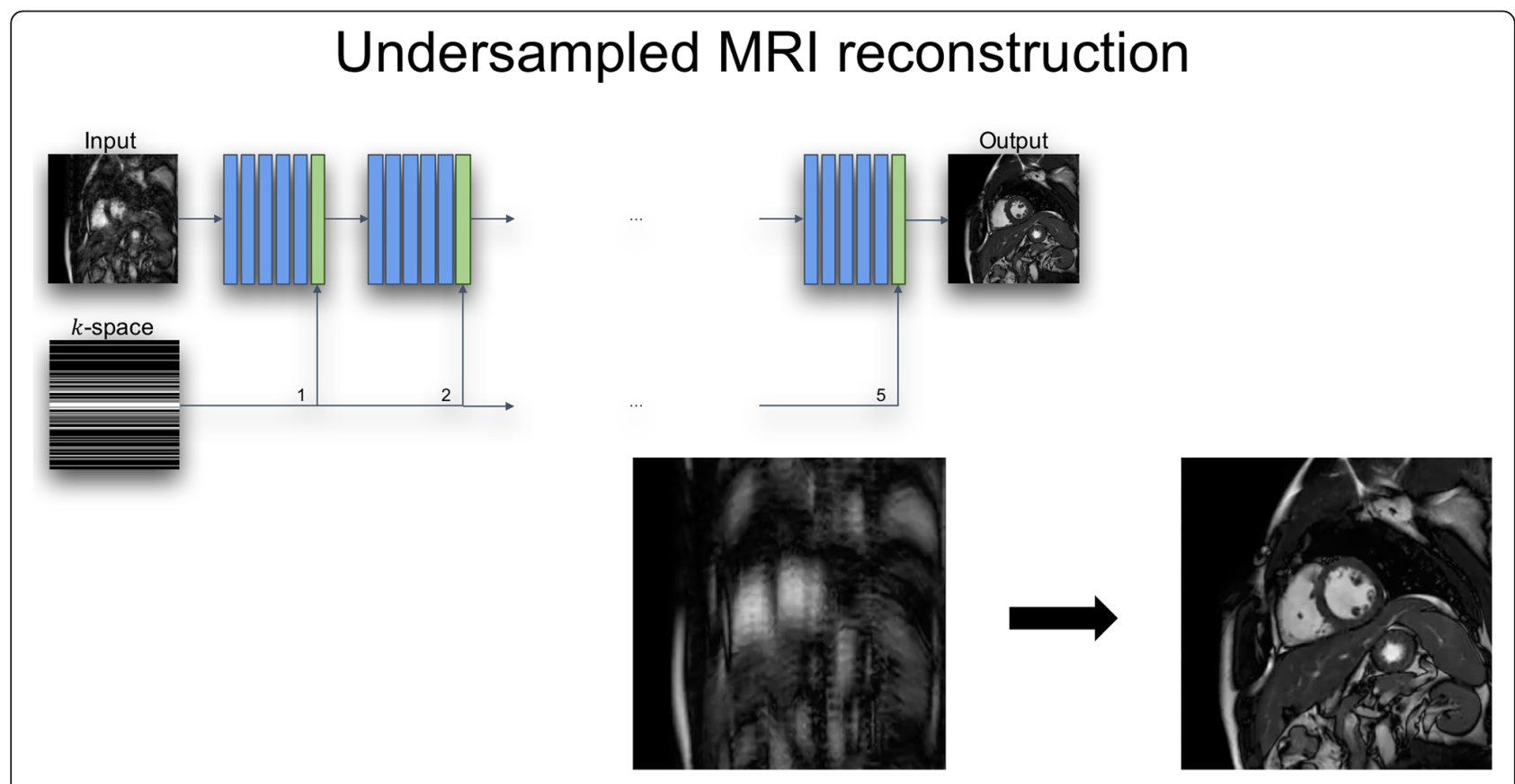

Fig. 3 Deep learning network for reconstruction of undersampled CMR images 


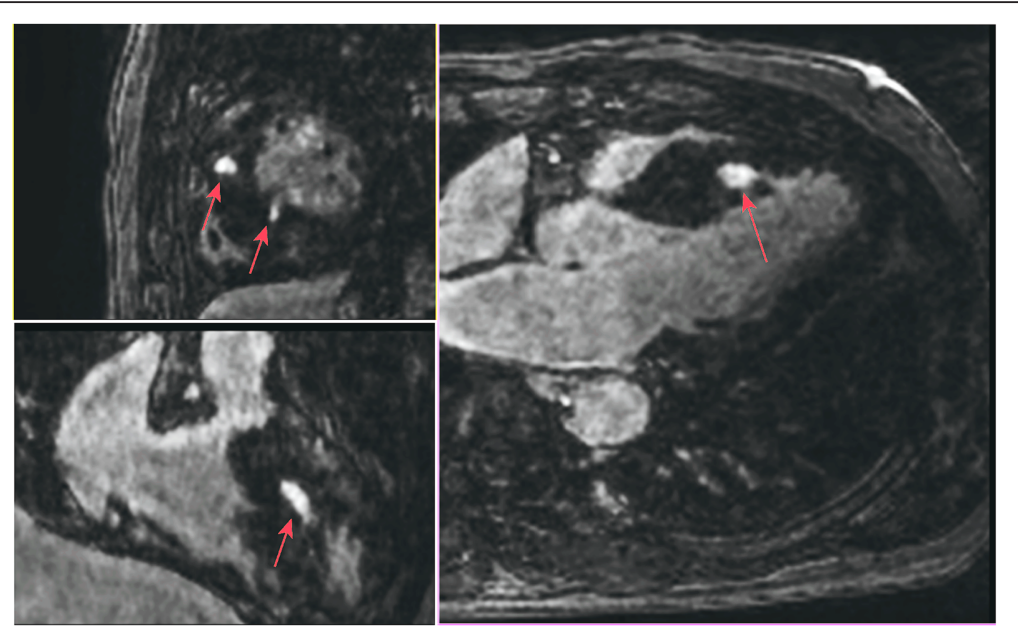

Fig. 4 Late gadolinium enhancement (LGE; red arrows) images with isotropic spatial resolution of $1.4 \mathrm{~mm}^{3}$ reconstructed using deep learning from a prospectively five-fold randomly undersampled 3D LGE dataset in a patient with hypertrophic cardiomyopathy

are still known to occur [34]. A large body of research has been dedicated to developing automated CMR segmentation methods (see reviews in $[35,36]$ ), but manual corrections are still needed in the areas where there are lots of trabeculae, the LV outflow tract, apical slices, as well the right ventricle. ML algorithms can be very helpful to further automate this task to increase the productivity of CMR segmentation while improving accuracy and reproducibility [37] over the techniques described in the two reviews mentioned earlier $[35,36]$. In general, DL-based fully automated LV segmentations are highly accurate with 9 out of 10 recently developed methods [37] achieving Dice similarity coefficients of 0.95 or better.

DL frameworks developed for general image segmentation can be applied directly to segment the myocardium and cardiac chambers from CMR images, often by using pixel-based classification. Many reports have been based on the U-Net architecture [38]. For instance, a basic CNN layout with 9 convolutional layers and a single upsampling layer was used to segment short-axis CMR images [39]. A fully convolutional approach with a simpler upsampling path has been suggested by Bai et al. [40] and successfully applied for pixelwise segmentation of 4-chamber, 2 chamber and short axis CMR images in less than $1 \mathrm{~min}$. Contextual 3D spatial information can also be integrated in the $\mathrm{CNN}$ architecture by providing features learnt from adjacent slices [41] or detecting a canonical view before segmentation [42]. Several studies have combined $\mathrm{CNN}$ with other ML algorithms, such as constraining the optimization process by constraining the network with information about the shape of the heart [43] or using the output of the DL model as the initial template for a deformable model segmentation $[44,45]$.
A different approach in DL segmentation is to perform regression rather than pixel classification. In [46], a network was trained to automatically identify myocardium and detect the center of the cavity. Then another network was trained to estimate radii from the cavity center, producing smooth epicardial and endocardial contours. A similar approach was also proposed by [47], where a boundary regression was performed on both left and right ventricles on short-axis images producing contours instead of pixel classification. Examples of image segmentation based on DL are shown in Figs. 5 and 6.

DL methods can also calculate functional parameters from imaging, e.g. fully automated determination of LVEF, which subsequently can be used as a basis to triage patients into different disease categories using handcrafted features [35, 48]. Puyol-Antón et al. [49] have taken this approach a step further and used a database of CMR and cardiac ultrasonography images as well as clinical information to design a ML-based diagnostic algorithm that can fully automatically identify patients with dilated cardiomyopathy using a support vector machine.

\section{Myocardial tissue characterization}

ML has been applied to a variety of myocardial tissue characterization tasks. For example, scar volume from LGE CMR is a quantitative imaging biomarker with inherent prognostic information, where application of ML allows to overcome the need for subjective, timeconsuming and labor-intensive manual delineation currently used in routine clinical practice. Even when using the current thresholding techniques for LGE quantification, accuracy and reproducibility remain a major challenge due to variations among different CMR centers [50], variations in gadolinium kinetics, and the patchy, 


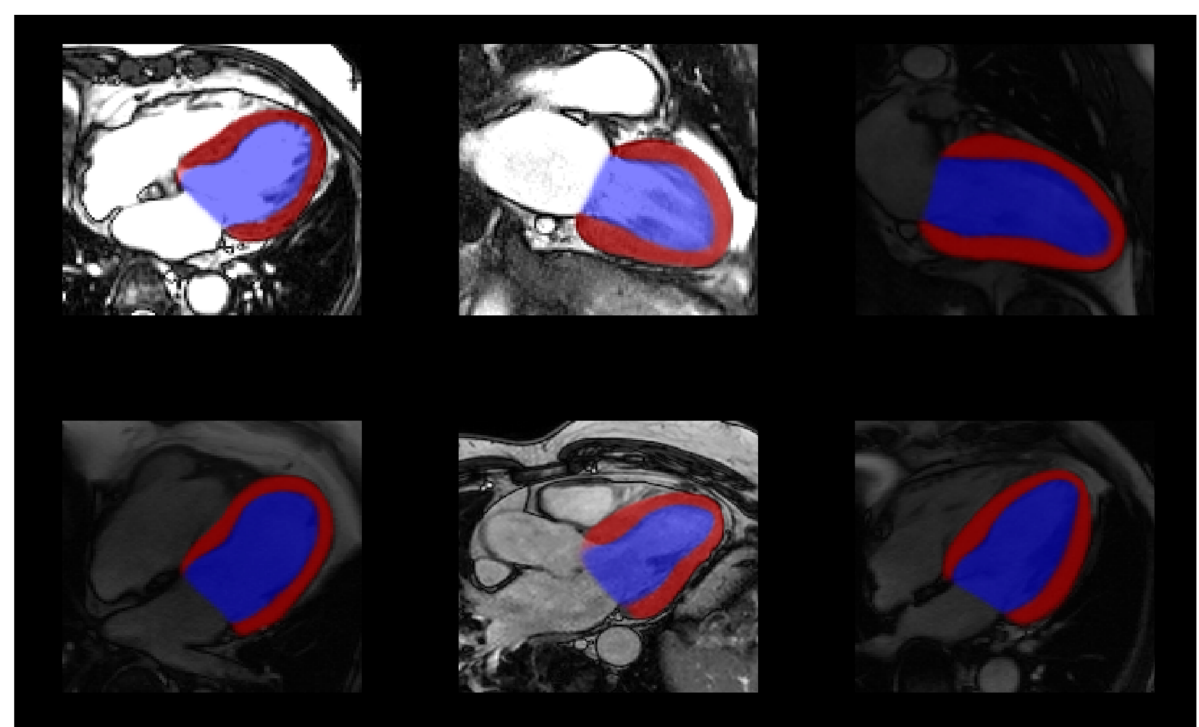

Fig. 5 Some examples of deep learning based myocardial segmentation on long-axis CMR images, trained from almost 5000 cases. A U-Net network architecture was used in this case to classify myocardium (red) and cavity (blue)

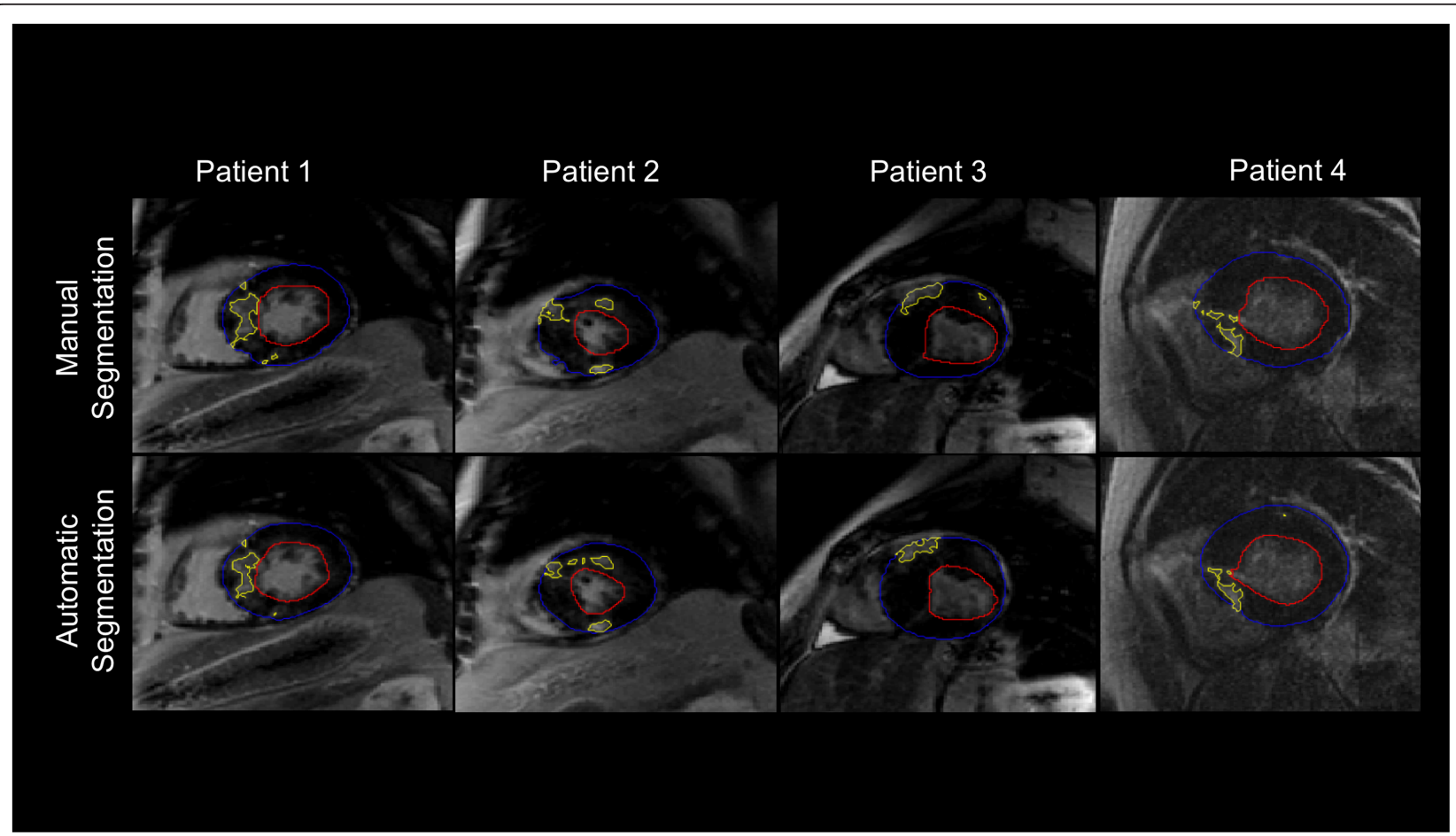

Fig. 6 Scar and myocardium segmentation results for slices from four different patients. Contours resulting from manual (top row) and automatic (lower row) segmentations for the epicardium (blue), endocardium (red), and scar (yellow) boundaries are overlaid on late gadolinium enhancement (LGE) images 
multifocal appearance of LGE, e.g. in patients with hypertrophic cardiomyopathy (HCM). To address these shortcomings, a novel, ML-based approach to LGE quantification has recently been suggested by Fahmy et al. [51].

Cardiac relaxometry has demonstrated capabilities for further quantitative tissue characterization. For example, T1 mapping has proven beneficial for the identification of diffuse myocardial fibrosis as well as myocardial edema and lipid deposition. While there have been numerous reports on data acquisition, little attention has been paid to data analysis and reporting. Recent work has shown that ML can also be applied to streamline data processing and analysis for myocardial tissue characterization [51-55] (Table 1 and Fig. 7).

The ability of ML techniques to cope with highdimensional data has recently facilitated the exponential growth of a novel field called radiomics. The term radiomics reflects a process of converting digital medical images into mineable high-dimensional data [56] by extracting a high number of handcrafted quantitative imaging features based on a wide range of mathematical and statistical methods. Various features can be extracted from images, the most important being morphologic, intensity-based, fractal-based, and texture features (subsumed under the term "texture analysis" [TA]) [57]. Texture features model spatial distributions of pixel grey levels and allow for the segmentation, analysis and classification of medical images according to the underlying tissue textures [58], thus offering the potential to overcome limitations of a pure visual image interpretation [59] (Fig.8).

Although radiomics and TA have been applied most prominently in the fields of oncologic and neurologic imaging, the first applications have been described for CMR. Since myocardial tissue characterization remains an important but complex and challenging task for differentiating amongst various cardiac diseases, the application of radiomics to CMR imaging data appears to be appealing in order to deliver further insights into the complex tissue changes and pathology of cardiovascular diseases.

The first applications of radiomics and TA in CMR have been reported for segmentation of scarred tissue areas in myocardial infarction [60-62], allowing for enhanced visualization of scarred myocardium and extracting information about the characteristics of the underlying myocardial tissue (Table2). Since then, several studies have been published, showing the feasibility of TA to differentiate between acute and chronic infarction [63], based either on a combination of non-contrast cine and LGE imaging [64], or based on cine imaging alone $[59,65,73]$.

Besides infarction, other applications of TA and radiomics have recently been reported for CMR. Several smaller studies demonstrated the use of texture features for differentiating amongst several causes of myocardial hypertrophy (i.e. HCM, amyloid and aortic stenosis) and healthy controls [74], or to detect fibrosis in HCM patients $[66,67]$. Cheng et al. [68] evaluated the prognostic value of texture features based on LGE imaging in HCM patients with systolic dysfunction, demonstrating that increased LGE heterogeneity was associated with adverse events in HCM patients with systolic dysfunction. Recently, TA has been applied to native T1mapping for discriminating between hypertensive heart disease and HCM patients, providing incremental value over global native T1 mapping [69].

Myocardial inflammation is another interesting topic, where radiomics and TA are extremely appealing in order to overcome the current limitations of qualitative as well as novel quantitative CMR sequences [11, 75]. Recent work has shown that averaging $\mathrm{T} 1$ and $\mathrm{T} 2$ values derived from $\mathrm{T} 1$ and $\mathrm{T} 2$ mapping over the entire myocardium has low sensitivity and specificity for detecting myocardial inflammation [11, 72, 75], and that analysis of inflammation-induced tissue inhomogeneity on T1 and/or T2 maps might enable more accurate quantification of myocardial inflammation [11, 71, 76]. Very recently, a first application of radiomics on $\mathrm{T} 1$ and

Table 1 Machine learning and deep learning for LGE quantification and parametric mapping

\begin{tabular}{|c|c|c|c|}
\hline Author & Myocardial disease & Image substrate & Application \\
\hline $\begin{array}{l}\text { Fahmy et al., } 2018 \\
\text { (Ref. 51) }\end{array}$ & $\mathrm{HCM}$ & LGE & Delineate and quantify scar volume in patients with $\mathrm{HCM}$ \\
\hline $\begin{array}{l}\text { Hann et al., } 2018 \\
\text { (Ref 52) }\end{array}$ & & T1 mapping & $\begin{array}{l}\text { Automated LV segmentation of T1 maps using a ShMOLLI sequence in order to } \\
\text { speed up LGE quantification based on T1 mapping }\end{array}$ \\
\hline $\begin{array}{l}\text { Fahmy et al., } 2019 \\
\text { (Ref 53) }\end{array}$ & Various diseases & T1 mapping & $\begin{array}{l}\text { DL based image analysis and motion correction for myocardial T1 mapping to } \\
\text { provide fast and automated T1 mapping analysis (DICE: } 0.85 \text { ) }\end{array}$ \\
\hline $\begin{array}{l}\text { Farrag et al., } 2019 \\
\text { (Ref 55) }\end{array}$ & Myocardial infarction & T1 mapping and CINE & $\begin{array}{l}\text { DL based automated LV segmentation of T1 maps using a ShMOLLI sequence } \\
\text { (DICE: 0.84) }\end{array}$ \\
\hline $\begin{array}{l}\text { Martini et al., } 2018 \\
\text { (Ref 54) }\end{array}$ & Various diseases & T1 mapping & Automated segmental analysis of T1 maps (DICE: 0.98, Jaccard: 0.97) \\
\hline
\end{tabular}

(Ref 54)

$M L$ machine learning, $D L$ deep learning, HCM hypertrophic cardiomyopathy, LGE late gadolinium enhancement, shMOLLI shortened modified Look-Locker inversion recovery 


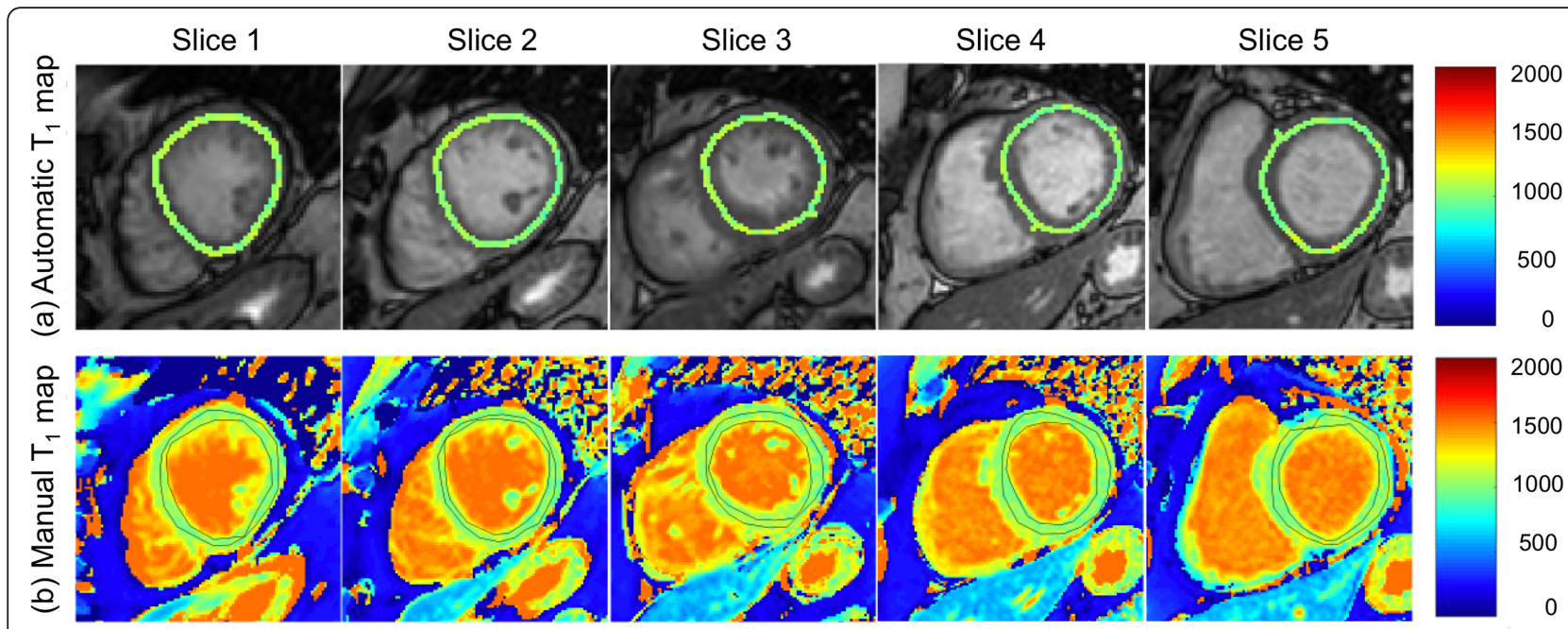

(c)

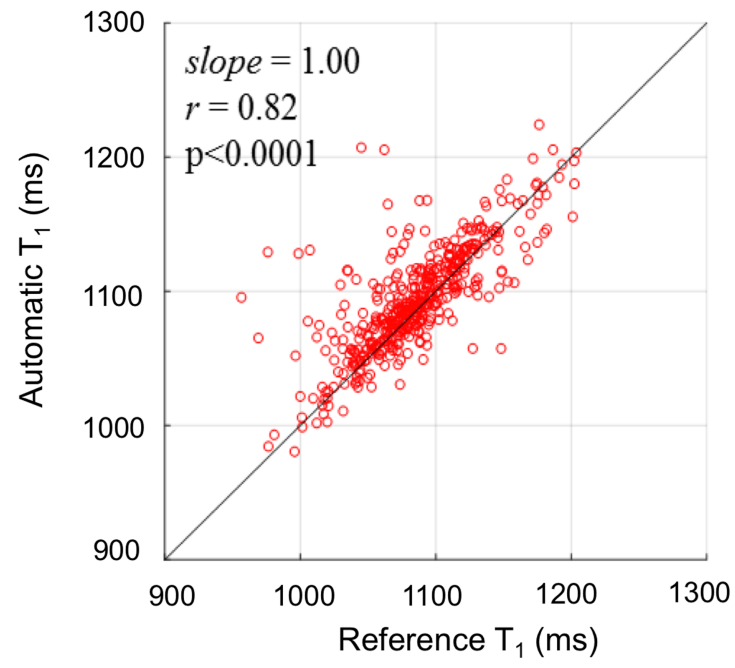

Per Slice

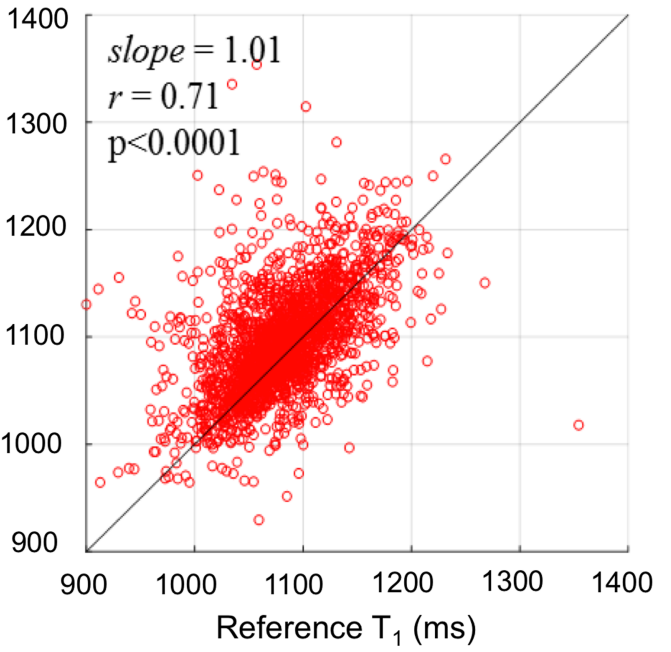

Fig. 7 Myocardial T1 mapping at five short axial slices (apex to base from left to right respectively) of the left ventricle of one patient. a Automatically reconstructed map (after automatic removal of myocardial boundary pixels) overlaid on a T1 weighted image with shortest inversion time; (a) Manually reconstructed T1 map. The contours in (b) represent the myocardium region of interest manually selected by the reader. In Fig. c scatter plots are shown of the automatic versus manual myocardium T1 values averaged over the patient volume (left) and each imaging slice (right). Solid lines represent the unity slope line

T2 mapping in a cohort of patients with biopsy-proven acute infarct-like myocarditis has demonstrated an excellent diagnostic accuracy of TA [70] and the concept has also been shown to be applicable to the much more challenging diagnosis of chronic myocardial inflammation or myocarditis presenting with heart-failure symptoms [77].

\section{Prognosis}

Information in CMR images obtained for diagnostic purposes can also be used for prognosis. In a meta-analysis of 56 studies containing data of 25,497 patients with suspected or known coronary artery disease (CAD) or recent myocardial infarction, El Aidi et al. [78] found that LVEF was an independent predictor of future cardiovascular events; predictors for patients with suspected or known coronary artery disease (CAD) were wall motion abnormalities, inducible perfusion defects, LVEF, and presence of infarction. Although meta-analyses such as these can help identify imaging features important for prognosis, selection of potentially relevant features is a manual process based on presumed pathophysiological importance and the ability to easily and reproducibly quantify parameters of interest. Another important limitation is that 'traditional' meta-analysis often fails to capture the heterogeneity between studies and patients in sufficient detail to establish the association between CMR findings and outcome. Furthermore, in almost all 


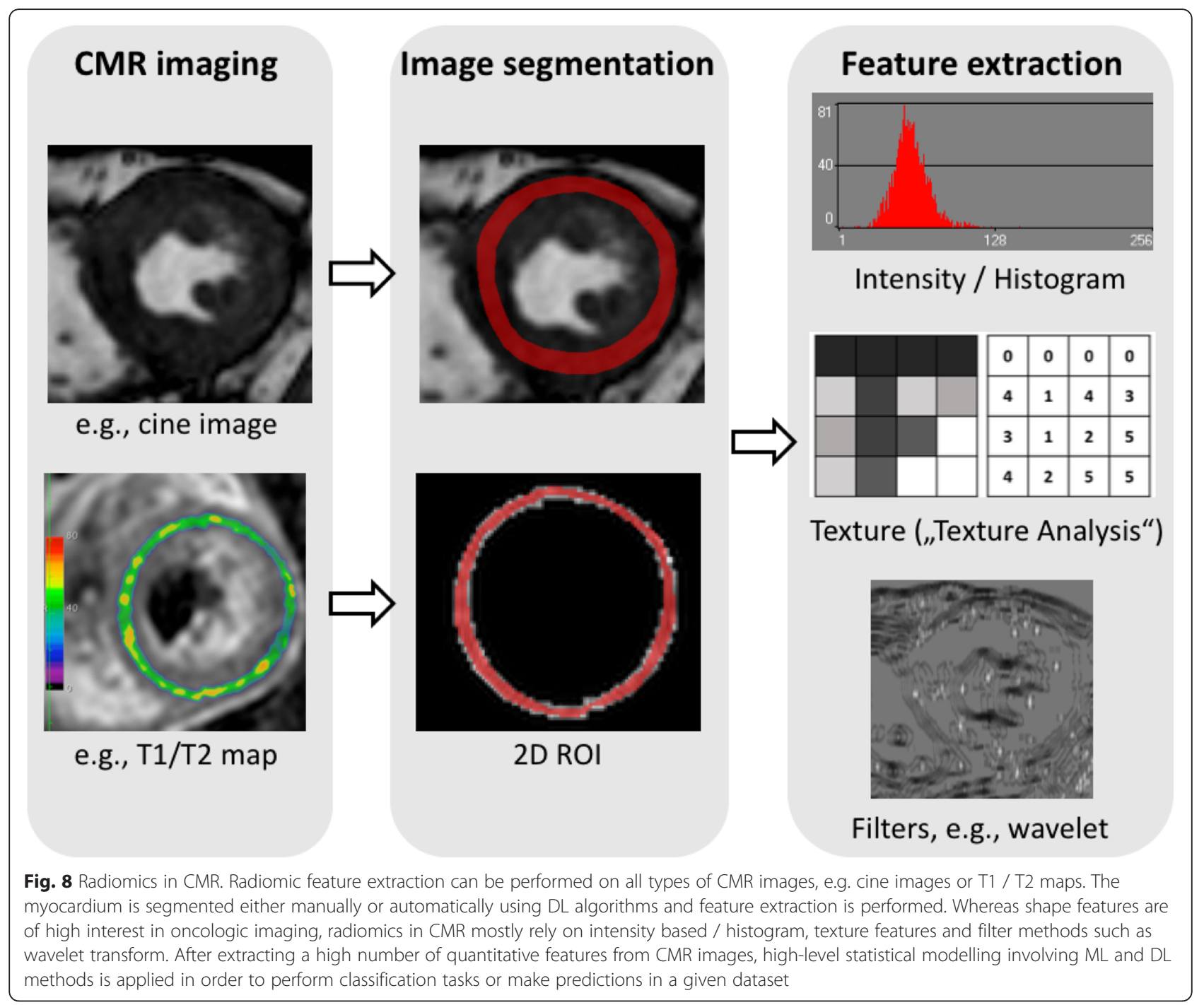

studies just a small fraction of all available information about individual patients is taken into account. Machine learning, on the other hand, is ideally suited in finding intrinsic structure within patient phenotypic data containing a high number (i.e. hundreds or even thousands) of variables, which can then be evaluated both retrospectively and prospectively for predicting outcomes. Thus, ML is much better suited for dealing with the high-dimensionality of 'real-world' datasets and can be used for unbiased identification of prognostically important variables. ML is also ideally suited to incorporate information from electronic health records (EHR), laboratory data and genetic analyses.

Machine learning for prediction of adverse cardiovascular events has the potential to augment of traditional risk scores, developed from the Framingham Heart Study and other large cohort studies, with novel biomarkers derived from imaging methods. In the Multi-Ethnic Study of Atherosclerosis (MESA), 6814 initially asymptomatic participants were followed for over 12 years. Over 700 variables were collected. Ambale-Venkatesh et al. [79] used a random survival forests technique to identify the top-20 predictors of each outcome measure. In addition to carotid ultrasound as a predictor for stroke, and coronary calcium score as a predictor of atherosclerotic cardiovascular disease, CMR derived LV structure and function were among the top predictors for incident heart failure [79]. The random survival forest risk prediction performed better than established risk scores with increased prediction accuracy. Another application is in dimension reduction methods, which show promise in the detection of multidimensional shape features to characterize ventricular remodeling. Mass, volume and univariate measures such as sphericity have shown prognostic value in MESA [80]. Zhang et al. [81] applied information maximizing component analysis to determine shape features which best characterize differences between patients with myocardial infarction and asymptomatic volunteers. 
Table 2 Radiomics and texture analysis in CMR

\begin{tabular}{|c|c|c|c|}
\hline Author & Myocardial disease & Image substrate & Application \\
\hline $\begin{array}{l}\text { Beliveau, P. et al., } 2015 \\
\text { (Ref 63) }\end{array}$ & $\begin{array}{l}\text { Myocardial fibrosis } \\
\text { (rat model) }\end{array}$ & LGE & $\begin{array}{l}\text { Detection of age-related myocardial fibrosis (correlation to } \\
\text { histopathology) }\end{array}$ \\
\hline $\begin{array}{l}\text { Engan, K. et al., } 2010 \\
\text { (Ref 60) }\end{array}$ & Myocardial infarction & LGE & $\begin{array}{l}\text { Discrimination of patients with low risk of arrhythmias from } \\
\text { those with high risk of arrhythmias }\end{array}$ \\
\hline $\begin{array}{l}\text { Kotu, L.P. et al., } 2013 \\
\text { (Ref 61) }\end{array}$ & Myocardial infarction & LGE & Automated segmentation of scarred tissue areas \\
\hline $\begin{array}{l}\text { Kotu, L.P. et al., } 2013 \\
\text { (Ref 62) }\end{array}$ & Myocardial infarction & LGE & Enhanced visualization and segmentation of scarred myocardium \\
\hline $\begin{array}{l}\text { Larroza, A. et al., } 2017 \\
\text { (Ref 64) }\end{array}$ & Myocardial infarction & LGE, Cine & $\begin{array}{l}\text { Differentiation between acute and chronic MI (AUC } 0.86 \text { for LGE, } \\
0.82 \text { for Cine) }\end{array}$ \\
\hline $\begin{array}{l}\text { Baeßler, B. et al., } 2018 \\
\text { (Ref 53) }\end{array}$ & Myocardial infarction & Cine & $\begin{array}{l}\text { Differentiation between normal myocardium and small (AUC 0.92) } \\
\text { as well as large scar (AUC 0.93) }\end{array}$ \\
\hline $\begin{array}{l}\text { Larroza, A. et al., } 2018 \\
\text { (Ref 65) }\end{array}$ & Myocardial infarction & Cine & $\begin{array}{l}\text { Differentiation between nonviable, viable, and remote myocardial } \\
\text { segments; extraction of TA features over the entire cardiac cycle; } \\
\text { AUC } 0.85\end{array}$ \\
\hline $\begin{array}{l}\text { Schofield, R. et al., } \\
2016 \text { (Ref 66) }\end{array}$ & $\begin{array}{l}\text { Hypertrophic heart (hypertrophic } \\
\text { cardiomyopathy, amyloid, aortic } \\
\text { stenosis) }\end{array}$ & Cine & $\begin{array}{l}\text { Differentiation amongst several causes of myocardial hypertrophy } \\
\text { (HCM, amyloid and aortic stenosis) and healthy controls }\end{array}$ \\
\hline $\begin{array}{l}\text { Thornhill, R.E. et al., } \\
2014 \text { (Ref 67) }\end{array}$ & Hypertrophic cardiomyopathy & LGE & $\begin{array}{l}\text { Differentiation between segments with and without hypertrophy } \\
\text { and fibrosis }\end{array}$ \\
\hline $\begin{array}{l}\text { Baeßler, B. et al., } 2018 \\
\text { (Ref 68) }\end{array}$ & Hypertrophic cardiomyopathy & Native T1-weighted & Differentiation between HCM patients and controls (AUC 0.95) \\
\hline $\begin{array}{l}\text { Cheng, S. et al., } 2018 \\
\text { (Ref 69) }\end{array}$ & Hypertrophic cardiomyopathy & LGE & $\begin{array}{l}\text { Association of adverse events in HCM patients with systolic } \\
\text { dysfunction with increased LGE heterogeneity }\end{array}$ \\
\hline $\begin{array}{l}\text { Neisius, U. et al., } 2018 \\
\text { (Ref } 70 \text { ) }\end{array}$ & $\begin{array}{l}\text { Hypertensive heart disease, } \\
\text { hypertrophic cardiomyopathy }\end{array}$ & Native T1 map & $\begin{array}{l}\text { Discrimination between hypertensive heart disease and HCM } \\
\text { patients with incremental value over global native T1 mapping }\end{array}$ \\
\hline $\begin{array}{l}\text { Baeßler, B. et al., } 2018 \\
\text { (Ref 71) }\end{array}$ & Acute myocarditis & $\begin{array}{l}\text { Native T1 mapping, } \\
\text { T2 mapping }\end{array}$ & $\begin{array}{l}\text { Diagnosis of biopsy-proven acute infarctlike myocarditis } \\
\text { (AUC 0.88) }\end{array}$ \\
\hline $\begin{array}{l}\text { Baeßler, B. et al., } 2017 \\
\text { (Ref 72) }\end{array}$ & $\begin{array}{l}\text { Dilated cardiomyopathy-like } \\
\text { myocarditis }\end{array}$ & $\begin{array}{l}\text { Native T1 mapping, } \\
\text { T2 mapping }\end{array}$ & $\begin{array}{l}\text { Diagnosis of biopsy-proven acute myocarditis presenting with } \\
\text { symptoms of heart failure }\end{array}$ \\
\hline
\end{tabular}

ML has also been used to predict outcome in patients with cardiovascular disease. In a small proof-of-concept study, Kotu et al. [82] used supervised ML to predict the occurrence of cardiac arrhythmia in patients who survived infarction. The investigators found that CMRderived scar texture features based on scar gradient and local binary patterns along with information about size and location of the scar demonstrated discriminative power for risk stratification comparable to currently used criteria such as LVEF and scar size. Finally, Bello et al. [83] used CMR-derived features together with clinical information to train a DL classifier that can predict outcome in patients with pulmonary hypertension. A dense motion model was used to identify patterns of right ventricular (RV) motion associated with adverse outcomes, with superior results to prognostication based on RV ejection fraction or strain.

ML methods have also been used to quantify relationships between cardiac morphology and genetic variations. For example, Schafer et al. found associations between titin-truncating variants and concentric remodeling in healthy individuals [84]. Mass univariate models were used to find associations with single nucleotide polymorphisms (SNPs) in genome-wide association studies (GWAS) studies [85]. Work by Peressutti et al. [86] has shown that ML could be used to identify patients with a favorable response to cardiac resynchronization therapy (CRT) by supervised learning of relationships between cardiac motion abnormalities, EKG data, clinical information and the success of CRT as assessed at 6-months follow-up. The same group of investigators has also demonstrated that more detailed analysis of myocardial strain at multiple different anatomical scales can be used to refine prediction of CRT response [87].

\section{Barriers to implementation}

Although ML and DL are powerful new methods which can help optimize the entire CMR imaging value chain, there are also several limitations that need to be mentioned. The most important present limitations include difficulty with rare entities and rare presentations of common entities such as congenital heart disease. Other difficulties include reliance on small, fixed inputs with long-range or heterogenous dependencies such as 
medical charts, prior exams, and dealing with multiple CMR imaging sequences and acquisition planes. Another limitation is the 'black-box' nature of DL algorithms since it is often unclear what information is used to come to a certain classification or result. Techniques to visualize salient features can potentially help address this limitation [88]. Furthermore, the present lack of model robustness and lack of portability with respect to different CMR scanners, sequences, imaging parameters and institutions need to be addressed. Another barrier in this regard is the lack of large, publicly available CMR datasets that can be used to objectively compare different (commercially available) algorithms with regard to their performance. Finally, many current ML and DL techniques are susceptible to adversarial attacks that may lead to erroneous results [89].

\section{Conclusions and future outlook}

$\mathrm{ML}$, and DL in particular, is beginning to be applied to different types of cardiac imaging [90]. Besides image interpretation, there are many tasks in the imaging process that can potentially benefit from application of ML. In the short term, ML techniques are highly likely to be incorporated in the image acquisition and reconstruction domains, in the postprocessing workflow and analysis of advanced image features beyond visually identifiable features as well as multi-dimensional contrasts and their interpretation. One promising method is to use DL methods to simulate images, both to augment the size and the variability in the training datasets for segmentation and classification networks and to characterize bias between different imaging modalities. A CMR scar simulation method has recently shown to improve identification of scar in LGE images [91]. Another promising technique is reinforcement learning, in which an agent is trained by trial and error using feedback from previous actions and experiences [92]

Despite the significant advances as described above there are currently no published clinical trials in which ML has been compared with human evaluation of CMR datasets. Prospective controlled clinical trials are required to establish the effectiveness of algorithms in clinical practice. The recently commenced CarDiac MagnEtic Resonance for Primary Prevention Implantable CardioVerter DebrillAtor ThErapy (DERIVATE) international observational registry is a good example of such a study [93]. Furthermore, validation must be performed not only using data from the same cohort as was employed in the training, but also from other cohorts. In particular, algorithms must be validated with data from different centers and different acquisition devices. An efficient way of subsequently comparing the performance of different algorithms is through so-called challenges - competitions where research teams evaluate their algorithms on a common dataset labeled with ground truth information, e.g. Kaggle platform [94] and grand challenge platform [95]. Ground truth also must be meticulously reviewed, in particular clinical reports since clinicians may disagree in reporting style (and findings) from center to center. Reported metrics are application dependent but need to include not only sensitivity and specificity but also positive predictive value and model metrics such as the area under the receiver operating characteristic curve. For the field to advance, algorithms should be published using open source repositories to enable replication, benchmarking, and improvement by other groups.

\section{Supplementary information}

Supplementary information accompanies this paper at https://doi.org/10. 1186/s12968-019-0575-y.

Additional file 1: Glossary of commonly used terms in Machine Learning. (DOCX $23 \mathrm{~kb}$ )

\section{Abbreviations \\ Al Artificial intelligence BOLD: Blood oxygen level dependent; CAD Coronary artery disease CMR: Cardiovascular magnetic resonance; CNN: Convolutional neural network; CRT: Cardiac resynchronization therapy; CS: Compressed sensing; DL: Deep learning; ED: End-diastolic; EHR: Electronic health records; ES: End-systolic; GLNU: Gray-level non-uniformity; \\ GPU: Graphics processing unit; GWAS: Genome-wide association study; HCM: Hypertrophic cardiomyopathy; LGE: Late gadolinium enhancement; LV Left ventricular LVEF: Left ventricular ejection fraction; MESA: Multi-ethnic Study of Atherosclerosis; MI: Myocardial infarction; ML: Machine learning; RLNU: Run-length non-uniformity; RV Right ventricular ShMOLLI: Shortened modified Look Locker inversion recovery; SNP: Single nucleotide polymorphism; TA: Texture analysis}

Acknowledgements

Not applicable.

Authors' contributions

All authors conceived of the review paper, participated in its design and coordination and helped to draft the manuscript. All authors read and approved the final manuscript.

\section{Funding}

EPSRC Programme Grant (EP/P001009/1) / Prof. Daniel Rueckert. Health Research Council of New Zealand (17/234) / Prof. Alistair Young. National Institutes of Health (US) (RO1HL121754) / Prof. Alistair Young. Netherlands Organization for Scientific Research (NWO) (P15-26) / Dr. Ivana Isgum.

Netherlands Organisation for Scientific Research (NWO) (P15-26) / Prof. Tim Leiner.

National Institutes of Health (US) (1R01HL129185, 1R01HL129157, R01HL127017).

Dr. Reza Nezafat.

American Heart Association (15EIA22710040) / Dr. Reza Nezafat.

Availability of data and materials

Please contact author for data requests.

Ethics approval and consent to participate

Not applicable.

Consent for publication

Not applicable. 


\section{Competing interests}

Dr. Nezafat has issued and pending patents related to methods for improved cardiovascular MRI, received source code from Philips Healthcare, and receives patent royalties from Phillips Healthcare and Samsung Electronics. Drs. Leiner and disclosed institutional grants received from Pie Medical B.V.

\section{Author details}

'Department of Radiology | E.01.132, Utrecht University Medical Center, Heidelberglaan 100, 3584CX Utrecht, The Netherlands. ${ }^{2}$ Biomedical Image Analysis Group, Department of Computing, Imperial College, London, UK. ${ }^{3}$ Department of Anatomy and Medical Imaging, University of Auckland, Auckland, New Zealand. ${ }^{4}$ Department of Radiology, University Hospital of Cologne, Cologne, Germany. ${ }^{5}$ Institute of Clinical Radiology and Nuclear Medicine, University Medical Centre Mannheim, Medical Faculty Mannheim, Heidelberg University, Mannheim, Germany. ${ }^{6}$ Department of Medicine (Cardiovascular Division), Beth Israel Deaconess Medical Centre, Harvard Medical School, Boston, MA, USA. ${ }^{7}$ Image Sciences Institute, University Medical Center Utrecht, Utrecht, Netherlands. ${ }^{8}$ Department of Biomedical Engineering, King's College London, London, UK.

\section{Received: 12 October 2018 Accepted: 2 September 2019}

\section{Published online: 07 October 2019}

\section{References}

1. Krittanawong C, Zhang H, Wang Z, Aydar M, Kitai T. Artificial Intelligence in Precision Cardiovascular Medicine. J Am Coll Cardiol. 2017;69:2657-64.

2. Johnson KW, Torres Soto J, Glicksberg BS, Shameer K, Miotto R, Ali M, et al. Artificial Intelligence in Cardiology. J Am Coll Cardiol. 2018;71:2668-79.

3. Miller DD, Brown EW. Artificial intelligence in medical practice: the question to the answer? Am J Med. 2018;131:129-33.

4. Krittanawong $C$. The rise of artificial intelligence and the uncertain future for physicians. Eur J Inter Med. 2018;48:e13-4.

5. Deo RC. Machine learning in medicine. Circulation. 2015;132:1920-30.

6. Bengio Y, Courville A, Vincent P. Representation learning: a review and new perspectives. IEEE Trans Pattern Anal Mach Intell. 2013;35:1798-828.

7. LeCun Y, Bengio Y, Hinton G. Deep learning. Nature. 2015;521:436-44.

8. Litjens G, Kooi T, Bejnordi BE, Setio AAA, Ciompi F, Ghafoorian M, et al. A survey on deep learning in medical image analysis. Med Image Anal. 2017; 42:60-88.

9. Oksuz I, Mukhopadhyay A, Dharmakumar R, Tsaftaris SA. Unsupervised myocardial segmentation for cardiac BOLD. IEEE Trans Med Imaging. 2017; 36:2228-38.

10. Wang G, Zhang Y, Hegde SS, Bottomley PA. High-resolution and accelerated multi-parametric mapping with automated characterization of vessel disease using intravascular MRI. J Cardiovasc Magn Reson. 2017;19.

11. Baeßler B, Schaarschmidt F, Dick A, Stehning C, Schnackenburg B, Michels G, et al. Mapping tissue inhomogeneity in acute myocarditis: a novel analytical approach to quantitative myocardial edema imaging by T2-mapping. J Cardiovasc Magn Reson. 2015;17.

12. Kramer CM, Barkhausen J, Flamm SD, Kim RJ, Nagel E. Standardized cardiovascular magnetic resonance imaging (CMR) protocols, society for cardiovascular magnetic resonance: board of trustees task force on standardized protocols. J Cardiovasc Magn Reson. 2008;10.

13. Frick M, Paetsch I, den Harder C, Kouwenhoven M, Heese H, Dries S, et al. Fully automatic geometry planning for cardiac MR imaging and reproducibility of functional cardiac parameters. J Magn Reson Imaging. 2011;34:457-67.

14. Hayes C, Daniel D, Lu X, Jolly M-P, Schmidt M. Fully automatic planning of the long-axis views of the heart. J Cardiovasc Magn Reson. 2013;15.

15. Goldfarb, JW, Cheng, J, Cao, JJ: Automatic optimal frequency adjustment for high field cardiac MR imaging via deep learning. In: CMR 2018 - A Joint EuroCMR/SCMR Meeting Abstract Supplement, pp. 437-438 (2018).

16. Jiang, W, Addy, O, Overall, W, Santos, J, Hu, B: Automatic artifacts detection as operative scan-aided tool in an autonomous MRI environment. In: CMR 2018 - A Joint EuroCMR/SCMR Meeting Abstract Supplement, pp. 11671168 (2018).

17. Kido T, Kido T, Nakamura M, Watanabe K, Schmidt M, Forman C, et al. Compressed sensing real-time cine cardiovascular magnetic resonance: accurate assessment of left ventricular function in a single-breath-hold. J Cardiovasc Magn Reson. 2016;18:50.
18. Chen X, Yang Y, Cai X, Auger DA, Meyer CH, Salerno M, et al. Accelerated twodimensional cine DENSE cardiovascular magnetic resonance using compressed sensing and parallel imaging. J Cardiovasc Magn Reson. 2016;18.

19. Axel L, Otazo R. Accelerated MRI for the assessment of cardiac function. Br J Radiol. 2016;89:20150655.

20. Vasanawala SS, Alley MT, Hargreaves BA, Barth RA, Pauly JM, Lustig M. Improved pediatric MR imaging with compressed sensing. Radiology. 2010; 256:607-16.

21. Akçakaya M, Basha TA, Chan RH, Manning WJ, Nezafat R. Accelerated isotropic sub-millimeter whole-heart coronary MRI: compressed sensing versus parallel imaging. Magn Reson Med. 2014;71:815-22.

22. Hsiao A, Lustig M, Alley MT, Murphy MJ, Vasanawala SS. Evaluation of valvular insufficiency and shunts with parallel-imaging compressed-sensing 4D phase-contrast MR imaging with stereoscopic 3D velocity-fusion volume-rendered visualization. Radiology. 2012;265:87-95.

23. Akçakaya M, Basha TA, Chan RH, Manning WJ, Nezafat R. Accelerated isotropic sub-millimeter whole-heart coronary MRI: compressed sensing versus parallel imaging: accelerated sub-millimeter whole-heart coronary MRI. Magn Reson Med. 2014;71:815-22.

24. Otazo R, Kim D, Axel L, Sodickson DK. Combination of compressed sensing and parallel imaging for highly accelerated first-pass cardiac perfusion MRI. Magn Reson Med. 2010;64:767-76.

25. Akçakaya M, Basha TA, Goddu B, Goepfert LA, Kissinger KV, Tarokh V, et al. Low-dimensional-structure self-learning and thresholding: regularization beyond compressed sensing for MRI reconstruction. Magn Reson Med. 2011;66:756-67.

26. Basha TA, Akçakaya M, Liew C, Tsao CW, Delling FN, Addae G, et al. Clinical performance of high-resolution late gadolinium enhancement imaging with compressed sensing. J Magn Reson Imaging. 2017:46:1829-38.

27. Caballero J, Price AN, Rueckert D, Hajnal JV. Dictionary learning and time sparsity for dynamic MR data reconstruction. IEEE Trans Med Imaging. 2014; 33:979-94.

28. Tsao J, Boesiger P, Pruessmann KP. K-t BLAST and k-t SENSE: dynamic MRI with high frame rate exploiting spatiotemporal correlations. Magn Reson Med. 2003;50:1031-42.

29. Jung H, Ye JC, Kim EY. Improved k-t BLAST and k-t SENSE using FOCUSS. Phys Med Biol. 2007;52:3201-26.

30. Hammernik K, Klatzer T, Kobler E, Recht MP, Sodickson DK, Pock T, et al. Learning a variational network for reconstruction of accelerated MRI data. Magn Reson Med. 2018;79:3055-71.

31. Schlemper J, Caballero J, Hajnal JV, Price AN, Rueckert D. A deep Cascade of convolutional neural networks for dynamic MR image reconstruction. IEEE Trans Med Imaging. 2018;37:491-503.

32. Qin C, Schlemper J, Caballero J, Price AN, Hajnal JV, Rueckert D. Convolutional recurrent neural networks for dynamic MR image reconstruction. IEEE Trans Med Imaging. 2019;38:280-90.

33. Schulz-Menger J, Bluemke DA, Bremerich J, Flamm SD, Fogel MA, Friedrich $M G$, et al. Standardized image interpretation and post processing in cardiovascular magnetic resonance: Society for Cardiovascular Magnetic Resonance (SCMR) Board of Trustees Task Force on standardized post processing. J Cardiovasc Magn Reson. 2013;15:35.

34. Suinesiaputra A, Bluemke DA, Cowan BR, Friedrich MG, Kramer CM, Kwong $R$, et al. Quantification of LV function and mass by cardiovascular magnetic resonance: multi-center variability and consensus contours. J Cardiovasc Magn Reson. 2015;17.

35. Petitjean C, Dacher J-N. A review of segmentation methods in short axis cardiac MR images. Med Image Anal. 2011;15:169-84.

36. Peng P, Lekadir K, Gooya A, Shao L, Petersen SE, Frangi AF. A review of heart chamber segmentation for structural and functional analysis using cardiac magnetic resonance imaging. MAGMA. 2016;29:155-95.

37. Bernard O, Lalande A, Zotti C, Cervenansky F, Yang X, Heng P-A, et al. Deep learning techniques for automatic MRI cardiac multi-structures segmentation and diagnosis: is the problem solved? IEEE Trans Med Imaging. 2018;37:2514-25

38. Ronneberger $\mathrm{O}$, Fischer $\mathrm{P}$, Brox T. U-net: convolutional networks for biomedica image segmentation. In: Navab N, Hornegger J, Wells WM, Frangi AF, editors. Medical image Computing and computer-assisted intervention - MICCAI 2015. Cham: Springer International Publishing; 2015. p. 234-41.

39. Romaguera LV, Romero FP, Fernandes Costa Filho CF, Fernandes Costa MG. Myocardial segmentation in cardiac magnetic resonance images using fully convolutional neural networks. Biomed Signal Process Control. 2018;44:48-57. 
40. Bai W, Sinclair M, Tarroni G, Oktay O, Rajchl M, Vaillant G, et al. Automated cardiovascular magnetic resonance image analysis with fully convolutional networks. J Cardiovasc Magn Reson. 2018;20.

41. Zheng Q, Delingette H, Duchateau N, Ayache N. 3-D consistent and robust segmentation of cardiac images by deep learning with spatial propagation. IEEE Trans Med Imaging. 2018;37:2137-48.

42. Vigneault DM, Xie W, Ho CY, Bluemke DA, Noble JA. $\Omega$-net (omega-net): fully automatic, multi-view cardiac MR detection, orientation, and segmentation with deep neural networks. Med Image Anal. 2018;48:95-106.

43. Oktay O, Ferrante E, Kamnitsas K, Heinrich M, Bai W, Caballero J, et al. Anatomically constrained neural networks (ACNNs): application to cardiac image enhancement and segmentation. IEEE Trans Med Imaging. 2018;37:384-95.

44. Avendi MR, Kheradvar A, Jafarkhani H. A combined deep-learning and deformable-model approach to fully automatic segmentation of the left ventricle in cardiac MRI. Med Image Anal. 2016;30:108-19.

45. Ngo TA, Lu Z, Carneiro G. Combining deep learning and level set for the automated segmentation of the left ventricle of the heart from cardiac cine magnetic resonance. Med Image Anal. 2017;35:159-71.

46. Tan LK, McLaughlin RA, Lim E, Abdul Aziz YF, Liew YM. Fully automated segmentation of the left ventricle in cine cardiac MRI using neural network regression: automated segmentation of LV. J Magn Reson Imaging. 2018;48: $140-52$.

47. Du X, Zhang W, Zhang H, Chen J, Zhang Y, Claude Warrington J, et al. Deep regression segmentation for cardiac bi-ventricle MR images. IEEE Access. 2018;6:3828-38

48. Wolterink JM, Leiner T, Viergever MA, Išgum I. Automatic Segmentation and Disease Classification Using Cardiac Cine MR Images. In: Pop M, Sermesant M, Jodoin P-M, Lalande A, Zhuang X, Yang G, et al., editors. Statistical Atlases and Computational Models of the Heart. ACDC and MMWHS Challenges. Cham: Springer International Publishing; 2018. p. 101-10.

49. Puyol-Anton E, Ruijsink B, Gerber B, Amzulescu MS, Langet H, De Craene M, et al. Regional multi-view learning for cardiac motion analysis: application to identification of dilated cardiomyopathy patients. IEEE Trans Biomed Eng. 2019;66:956-66.

50. Engblom H, Tufvesson J, Jablonowski R, Carlsson M, Aletras AH, Hoffmann $P$, et al. A new automatic algorithm for quantification of myocardial infarction imaged by late gadolinium enhancement cardiovascular magnetic resonance: experimental validation and comparison to expert delineations in multi-center, multi-vendor patient data. J Cardiovasc Magn Reson. 2016;18:27.

51. Fahmy AS, Rausch J, Neisius U, Chan RH, Maron MS, Appelbaum E, et al. Automated cardiac MR scar quantification in hypertrophic cardiomyopathy using deep convolutional neural networks. JACC Cardiovasc Imaging. 2018; 11:1917-8

52. Hann, E, Ferreira, VM, Neubauer, S, Piechnik, SK: Deep learning for fully automatic contouring of the left ventricle in cardiac T1 mapping. In: CMR 2018 - A Joint EuroCMR/SCMR Meeting Abstract Supplement, pp. 401-402 (2018).

53. Fahmy AS, El-Rewaidy H, Nezafat M, Nakamori S, Nezafat R. Automated analysis of cardiovascular magnetic resonance myocardial native T1 mapping images using fully convolutional neural networks. J Cardiovasc Magn Reson. 2019;21.

54. Martini, N, Latta, DD, Santini, G, Valvano, G, Barison, A, Susini, CL, et al.: A deep learning approach for the segmental analysis of myocardial T1 mapping. In: CMR 2018 - A Joint EuroCMR/SCMR Meeting Abstract Supplement, pp. 593-594 (2018).

55. Farrag NA, White JA, Ukwatta E. Semi-automated myocardial segmentation of T1-mapping cardiovascular magnetic resonance images using deformable non-rigid registration from CINE images. In: Gimi B, Krol A, editors. Medical Imaging 2019: Biomedical applications in molecular, structural, and functional imaging. San Diego, United States: SPIE; 2019. p. 46.

56. Gillies RJ, Kinahan PE, Hricak H. Radiomics: images are more than pictures. They Are Data Radiology. 2016;278:563-77

57. Vallières M, Zwanenburg A, Badic B, Chezelerest C, Visvikis D, Hatt M. Responsible Radiomics research for faster clinical translation. J Nucl Med 2018:59:189-193.

58. Tourassi GD. Journey toward computer-aided diagnosis: role of image texture analysis. Radiology. 1999;213:317-20.

59. Baeßler B, Mannil M, Oebel S, Maintz D, Alkadhi H, Manka R. Subacute and chronic left ventricular myocardial scar: accuracy of texture analysis on nonenhanced cine MR images. Radiology. 2018;286:103-12.
60. Engan K, Eftestøl T, Ørn S, Kvaløy JT, Woie L. Exploratory data analysis of image texture and statistical features on myocardium and infarction areas in cardiac magnetic resonance images. In: 2010 Annual International Conference of the IEEE Engineering in Medicine and Biology. Buenos Aires: IEEE; 2010. p. 5728-31.

61. Kotu LP, Engan K, Skretting K, Måløy F, Orn S, Woie L, et al. Probability mapping of scarred myocardium using texture and intensity features in CMR images. Biomed Eng Online. 2013;12:91.

62. Kotu LP, Engan K, Skretting K, Ørn S, Woie L, Eftestøl T. Segmentation of scarred myocardium in cardiac magnetic resonance images. ISRN Biomed Imaging. 2013;2013:1-12.

63. Beliveau P, Cheriet F, Anderson SA, Taylor JL, Arai AE, Hsu L-Y. Quantitative assessment of myocardial fibrosis in an age-related rat model by ex vivo late gadolinium enhancement magnetic resonance imaging with histopathological correlation. Comput Biol Med. 2015;65:103-13.

64. Larroza A, Materka A, López-Lereu MP, Monmeneu JV, Bodí V, Moratal D. Differentiation between acute and chronic myocardial infarction by means of texture analysis of late gadolinium enhancement and cine cardiac magnetic resonance imaging. Eur J Radiol. 2017;92:78-83.

65. Zhang N, Yang G, Gao Z, Xu C, Zhang Y, Shi R, et al. Deep Learning for Diagnosis of Chronic Myocardial Infarction on Nonenhanced Cardiac Cine MRI. Radiology. 2019;291:606-617.

66. Thornhill RE, Cocker M, Dwivedi G, Dennie C, Fuller L, Dick A, et al. Quantitative texture features as objective metrics of enhancement heterogeneity in hypertrophic cardiomyopathy. J Cardiovasc Magn Reson. 2014;16(suppl 1):P351.

67. Baeßler B, Mannil M, Maintz D, Alkadhi H, Manka R. Texture analysis and machine learning of non-contrast T1-weighted MR images in patients with hypertrophic cardiomyopathy_-preliminary results. Eur J Radiol. 2018;102:61-7.

68. Cheng S, Fang M, Cui C, Chen X, Yin G, Prasad SK, et al. LGE-CMR-derived texture features reflect poor prognosis in hypertrophic cardiomyopathy patients with systolic dysfunction: preliminary results. Eur Radiol. 2018;28:4615-24.

69. Neisius U, El-Rewaidy H, Nakamori S, Rodriguez J, Manning WJ, Nezafat R. Radiomic analysis of myocardial native $\mathrm{T} 1$ imaging discriminates between hypertensive heart disease and hypertrophic cardiomyopathy. JACC: Cardiovascular Imaging. 2019.

70. Baeßler B, Luecke C, Lurz J, Klingel K, von Roeder M, de Waha S, et al. Cardiac MRI texture analysis of T1 and T2 maps in patients with Infarctlike acute myocarditis. Radiology. 2018;289:357-65.

71. Baeßler B, Treutlein M, Schaarschmidt F, Stehning C, Schnackenburg B, Michels $G$, et al. A novel multiparametric imaging approach to acute myocarditis using T2-mapping and CMR feature tracking. J Cardiovasc Magn Reson. 2017;19.

72. Bönner F, Spieker M, Haberkorn S, Jacoby C, Flögel U, Schnackenburg B, et al. Myocardial T2 mapping increases noninvasive diagnostic accuracy for biopsy-proven myocarditis. JACC Cardiovasc Imaging. 2016;9:1467-9.

73. Larroza A, López-Lereu MP, Monmeneu JV, Gavara J, Chorro FJ, Bodí V, et al. Texture analysis of cardiac cine magnetic resonance imaging to detect nonviable segments in patients with chronic myocardial infarction. Med Phys. 2018:45:1471-80.

74. Schofield R, Ganeshan B, Kozor R, Nasis A, Endozo R, Groves A, et al. CMR myocardial texture analysis tracks different etiologies of left ventricular hypertrophy. J Cardiovasc Magn Reson. 2016;18:082.

75. Lurz P, Luecke C, Eitel I, Föhrenbach F, Frank C, Grothoff M, et al. Comprehensive cardiac magnetic resonance imaging in patients with suspected myocarditis. J Am Coll Cardiol. 2016;67:1800-11.

76. Baeßler B, Schaarschmidt F, Treutlein M, Stehning C, Schnackenburg B, Michels $G$, et al. Re-evaluation of a novel approach for quantitative myocardial oedema detection by analysing tissue inhomogeneity in acute myocarditis using T2-mapping. Eur Radiol. 2017;27:5169-78.

77. Baeßler B, Luecke C, Klingel K, Kandolf R, Schuler G, Maintz D, et al. P2583Texture analysis and machine learning applied on cardiac magnetic resonance T2 mapping: incremental diagnostic value in biopsy-proven acute myocarditis. Eur Heart J. 2017;38(suppl_1):eh×502.P2583. https://doi. org/10.1093/eurheartj/eh×502.P2583.

78. El Aidi H, Adams A, Moons KGM, Den Ruijter HM, Mali WPTM, Doevendans PA, et al. Cardiac magnetic resonance imaging findings and the risk of cardiovascular events in patients with recent myocardial infarction or suspected or known coronary artery disease. J Am Coll Cardiol. 2014;63: $1031-45$. 
79. Ambale-Venkatesh B, Yang X, Wu CO, Liu K, Hundley WG, McClelland R, et al. Cardiovascular event prediction by machine learning: the multi-ethnic study of atherosclerosis. Circ Res. 2017;121:1092-101.

80. Ambale-Venkatesh B, Yoneyama K, Sharma RK, Ohyama Y, Wu CO, Burke GL, et al. Left ventricular shape predicts different types of cardiovascular events in the general population. Heart. 2017;103:499-507.

81. Zhang X, Ambale-Venkatesh B, Bluemke DA, Cowan BR, Finn JP, Kadish AH, et al. Information maximizing component analysis of left ventricular remodeling due to myocardial infarction. J Transl Med. 2015;13:343.

82. Kotu LP, Engan K, Borhani R, Katsaggelos AK, Ørn S, Woie L, et al. Cardiac magnetic resonance image-based classification of the risk of arrhythmias in post-myocardial infarction patients. Artif Intell Med. 2015;64:205-15.

83. Bello GA, Dawes TJW, Duan J, Biffi C, de Marvao A, Howard LSGE, et al. Deep learning cardiac motion analysis for human survival prediction. Nat Mach Intell. 2019:1:95-104.

84. Schafer S, de Marvao A, Adami E, Fiedler LR, Ng B, Khin E, et al. Titintruncating variants affect heart function in disease cohorts and the general population. Nat Genet. 2017:49:46-53.

85. Biffi C, de Marvao A, Attard MI, Dawes TJW, Whiffin N, Bai W, et al. Threedimensional cardiovascular imaging-genetics: a mass univariate framework. Bioinformatics. 2018;34:97-103.

86. Peressutti D, Sinclair M, Bai W, Jackson T, Ruijsink J, Nordsletten D, et al. A framework for combining a motion atlas with non-motion information to learn clinically useful biomarkers: application to cardiac resynchronisation therapy response prediction. Med Image Anal. 2017;35:669-84.

87. Sinclair M, Peressutti D, Puyol-Antón E, Bai W, Rivolo S, Webb J, et al. Myocardial strain computed at multiple spatial scales from tagged magnetic resonance imaging: estimating cardiac biomarkers for CRT patients. Med Image Anal. 2018;43:169-85.

88. Seah JCY, Tang JSN, Kitchen A, Gaillard F, Dixon AF. Chest radiographs in congestive heart failure: visualizing neural network learning. Radiology 2019;290:514-22.

89. Fawaz HI, Forestier G, Weber J, Idoumghar L, Muller P-A. Adversarial Attacks on Deep Neural Networks for Time Series Classification. arXiv:190307054 [cs, stat]. 2019. http://arxiv.org/abs/1903.07054. Accessed 8 Jun 2019.

90. Madani A, Arnaout R, Mofrad M, Arnaout R. Fast and accurate view classification of echocardiograms using deep learning. npj Digital Medicine. 2018;1.

91. Lau F, Hendriks T, Lieman-Sifry J, Norman B, Sall S, Golden D. ScarGAN: Chained Generative Adversarial Networks to Simulate Pathological Tissue on Cardiovascular MR Scans. arXiv:180804500 [cs]. 2018. http://arxiv.org/abs/1 808.04500. Accessed 8 Jun 2019.

92. Alansary A, Oktay O, Li Y, Folgoc LL, Hou B, Vaillant G, et al. Evaluating reinforcement learning agents for anatomical landmark detection. Med Image Anal. 2019;53:156-64.

93. Guaricci Al, Masci PG, Lorenzoni V, Schwitter J, Pontone G. CarDiac MagnEtic resonance for primary prevention implantable CardioVerter DebrillAtor ThErapy international registry: design and rationale of the DERIVATE study. Int J Cardiol. 2018;261:223-7.

94. Kaggle Inc.: Kaggle Data Science Platform. http://www.kaggle.com/. Last accessed 11 Sept 2019

95. Open Medical Image Computing: Grand-Challenges. https://grandchallenge.org/. Last accessed 11 Sept 2019.

\section{Publisher's Note}

Springer Nature remains neutral with regard to jurisdictional claims in published maps and institutional affiliations.

Ready to submit your research? Choose BMC and benefit from:

- fast, convenient online submission

- thorough peer review by experienced researchers in your field

- rapid publication on acceptance

- support for research data, including large and complex data types

- gold Open Access which fosters wider collaboration and increased citations

- maximum visibility for your research: over $100 \mathrm{M}$ website views per year

At BMC, research is always in progress.

Learn more biomedcentral.com/submissions 